\title{
Implicaciones de la Inspección Educativa en Cataluña en el Proceso de Autonomía de Centros
}

\section{Implications of the Educational Inspection in Catalonia in the Process of Autonomy of Schools}

\author{
Joan Segura ${ }^{1, *}$, Joaquín Gairín ${ }^{2}$, Patricia Silva ${ }^{1}$ \\ ${ }^{1}$ Universitat de Lleida, España \\ ${ }^{2}$ Universitat Autònoma de Barcelona, España
}

\section{DESCRIPTORES:}

Inspección educativa

Dirección escolar

Autonomía de centros

Calidad educativa

\begin{abstract}
RESUMEN:
Cataluña es un referente en autonomía de centros a partir de la aprobación de la Ley educativa (LEC) de 16 de julio de 2009. El presente estudio analiza los cambios en las funciones de la Inspección educativa en su relación e implicación con los centros y la dirección a partir del desarrollo del Decreto de autonomía de Centros (2010). El interés se centra en indagar en la Inspección educativa como unos de los actores principales en este proceso de autonomía. La metodología mixta, ha combinado aproximaciones cuantitativas y cualitativas y consideró la región como caso de estudio. El estudio ha incluido la aplicación de un cuestionario con una muestra final de 88 inspectores/as, 12 entrevistas en profundidad y 2 grupos de discusión de 5 inspectores/as y de 6 directores/ as de centros educativos. El estudio cuantitativo realiza un análisis descriptivo y el cualitativo profundiza en algunos datos y busca procesos interpretativos por parte de los implicados en la temática. Los resultados revelan el papel de la Inspección en el proceso de implementación de la autonomía en los centros, y como soporte a las Direcciones escolares. El estudio afirma que la Inspección debe evolucionar hacia una realidad más práctica y presencial, disminuyendo la burocracia y definiendo y seleccionando sus tareas y funciones.
\end{abstract}

\section{KEYWORDS:}

Educational inspection School leadership Autonomy of schools Educational quality

\section{ABSTRACT:}

Catalonia is a benchmark in the regulation of the autonomy of schools as of the approval of the Educational Law (LEC) of July 16, 2009. This study analyzes the changes in the functions of the Educational Inspection in its relationship and involvement with schools and the leadership from the development of the Schools Autonomy Decree (2010). The interest is focused on investigating the Educational Inspection as one of the main actors in this autonomy process. The mixed methodology has combined quantitative and qualitative approaches and considered the region as a case study. The field study has included the application of a questionnaire with a final sample of 88 inspectors, 12 indepth interviews and 2 discussion groups of 5 inspectors and 6 directors of schools. The quantitative study performs a descriptive analysis and the qualitative study delves into some data and looks for interpretive processes by those involved in the subject. The results reveal the role of the Inspectorate in the entire process of implementing autonomy in schools, and as a support for school directorates. The study stated that the Inspection must evolve towards a more practical and face-to-face reality, reducing the bureaucracy and defining and selecting its tasks and functions..

CÓMO CITAR:

Segura, J., Gairín, J. y Silva, P. (2021). Implicaciones de la inspección educativa en Cataluña en el proceso de autonomía de centros. REICE. Revista Iberoamericana sobre Calidad, Eficacia y Cambio en Educación, 19(1), 53-62. https://doi.org/10.15366/reice2021.19.1.004

${ }^{*}$ Contacto: j.segura@pip.udl.cat

ISSN: $1696-4713$

www.rinace.net/reice/

revistas.uam.es/reice
Recibido:

27 de marzo 2020

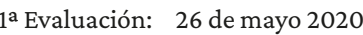

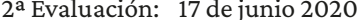

Aceptado: 13 de julio 2020 


\section{Introducción}

Según Moreno y otros (2018), en los últimos diez años se han producido un incremento de estudios referentes a la Inspección educativa debido al interés por parte de la comunidad científica y la aportación que supone en mundo educativo.

El presente estudio analiza los cambios que se están produciendo en las funciones de la Inspección educativa en Cataluña respecto a los centros educativos y sus direcciones a partir del desarrollo del Decreto de autonomía de Centros desarrollado a partir de la Ley educativa (LEC) aprobada el 16 de julio de 2009. Esta, supuso la concreción de dos aspectos fundamentales sobre organización y gestión educativa: el Decreto de autonomía de centros educativos y el Decreto de direcciones de centros públicos.

El Decreto 102/2010 de Autonomía de centros educativos nace con el objetivo de mejorar el rendimiento y los resultados educativos del alumnado y la cohesión social. El Decreto 155/2010, de 2 de noviembre, de Dirección de los centros docentes trata de fortalecer la figura del Director, otorgándole más competencias y más responsabilidades para liderar proyectos educativos sólidos para sus centros, asumiendo algunas tareas que hasta ahora eran competencia exclusiva de la Inspección educativa.

Las funciones de la Inspección educativa no varían ante estos cambios, pero si su relación con los centros educativos y los equipos directivos. Estos aspectos son los abordados por el estudio a partir de una metodología mixta que, combinando aproximaciones cuantitativas y cualitativas, se desarrolla en 2 fases: una primera descriptiva, y una segunda interpretativa.

\section{Marco teórico}

El informe de la OCDE (2014), a partir de las pruebas PISA de 2012, afirma que, los países donde los centros educativos rinden cuentas de sus resultados haciendo públicos los datos de sus logros y tienen una mayor autonomía en la asignación de los recursos, tienden a obtener un mejor rendimiento de los estudiantes que aquellos que cuentan con menos autonomía. Aporta unos datos y una de sus recomendaciones subraya que los centros españoles tienen menos autonomía que la media de la OCDE para el desarrollo de contenidos y la evaluación: en España, el 58\% de los alumnos asisten a centros con cierta autonomía para determinar su oferta educativa, comparado con el $82 \%$ de media en los países desarrollados (Segura, 2018).

En 1985, España, a través de la Ley Orgánica del Derecho a la Educación (LODE), comienza a fijar las bases para desarrollar la autonomía, pero no ha sido hasta el año 2006, aprobada la Ley Orgánica de Educación (LOE) y el 2013, a partir de la Ley Orgánica para la Mejora de la Calidad Educativa (LOMCE), cuando se ha reforzado el principio de autonomía de los centros educativos. Esta última Ley hace una apuesta muy fuerte por el desarrollo de la autonomía de centros, con el objetivo de combatir el fracaso escolar y mejorar el rendimiento académico de los alumnos a través de esta autonomía otorgada a los centros.

Según Bolívar (2010), la LOE fue la Ley clave para iniciar todo el proceso de autonomía a los centros, ya que abrió las puertas a las diferentes Comunidades Autonómicas para que pudieran desarrollarla en su territorio a través de los Reglamentos Orgánicos de los centros (Andalucía y otras comunidades) o del Decreto de Autonomía de centros (Cataluña).

El Decreto se autonomía de los centros de Catalunya es uno de los primeros del Estado Español y se completa con el Decreto de Direcciones (2010), que da respuesta a todas las necesidades que se cree carecían las direcciones de los centros escolares explicitando de manera muy clara las tareas, funciones, figura, temporalidad, formación, acceso de las direcciones u otras actividades de este rol.

Contrariamente, no se genera ningún cambio vinculado con las tareas de la Inspección educativa. Según Silva (2011), el Decreto 102/2010 de Autonomía únicamente señala que la Inspección de educación debe adecuar las actuaciones que le corresponden en el ejercicio de sus funciones al 
régimen de autonomía de los centros (art. 3.2.), lo mismo que el artículo 179.2 LEC (2009) cuando hace referencia a las atribuciones de la Inspección. Resumiendo, y en pocas palabras, la Inspección debe adaptarse a las Direcciones de los centros y a sus necesidades.

Actualmente en Cataluña, la Inspección educativa está regulada por el Decreto 266/2000, de 31 de julio, con la modificación de algún artículo en 2002, cuando todavía no era una realidad la autonomía de centros. Su artículo 2 hace referencia a las funciones de:

- Supervisar, asesorar, evaluar los centros y al profesorado

- Orientar a los equipos directivos

- Cuidar por el cumplimiento de la normativa

- Informar y colaborar en actividades vinculadas a la enseñanza.

Estas funciones van en consonancia con las que se desarrollan en diferentes países europeos. Tal como afirma Lorente (2010), considera que la Inspección europea lleva a cabo tres funciones básicas y propias como órgano de la administración. Estas son:

- Ejercer la función de control de la normativa legal como garantía del derecho de los ciudadanos a la educación.

- Realizar la evaluación del funcionamiento y de los resultados de los centros educativos y de sus profesores.

- Asesorar y orientar para una adecuada aplicación de la legislación educativa con el fin de una mayor calidad educativa.

Retornando a Cataluña, Del Pozo (2016a), en relación a estas funciones, hace referencia al nuevo papel de la Dirección escolar:

La LEC (2009), al definir las nuevas competencias del director de los centros públicos, acercó su perfil a lo que hasta entonces ostentaba sólo la Inspección educativa. Así, para que el director pudiera ejercer sus competencias como jefe de personal, la Ley lo consideró como autoridad pública, le asignó competencias para intervenir en la evaluación del personal del centro y capacidad para observar la práctica docente en el aula y, en general, para supervisar todas las actividades educativas. Hasta ese momento, estas cuatro competencias eran exclusivas de la inspección educativa. (p. 9)

Se apuesta por un proceso de cambio a partir de la autonomía de centros, pero no se acaban de adecuar las actuaciones de la Inspección en todo este estado de transformación. Posiblemente sea este uno de los mayores conflictos en el que se encuentra actualmente el Sistema educativo catalán y un problema que no ha sabido resolver o descifrar.

La figura 1 representa la situación actual de la Dirección escolar, que sale muy reforzada, ganando en competencias y responsabilidad y compartiendo funciones que hasta ahora eran exclusivas de la Inspección. Paralelamente, la Inspección, continúa rigiéndose por un Decreto del año 2000 y, por lo tanto, sin una actualización tan necesaria en el actual marco de autonomía. 
Figura 1

Mapa conceptual de las funciones y tareas entre dirección escolar e inspección educativa

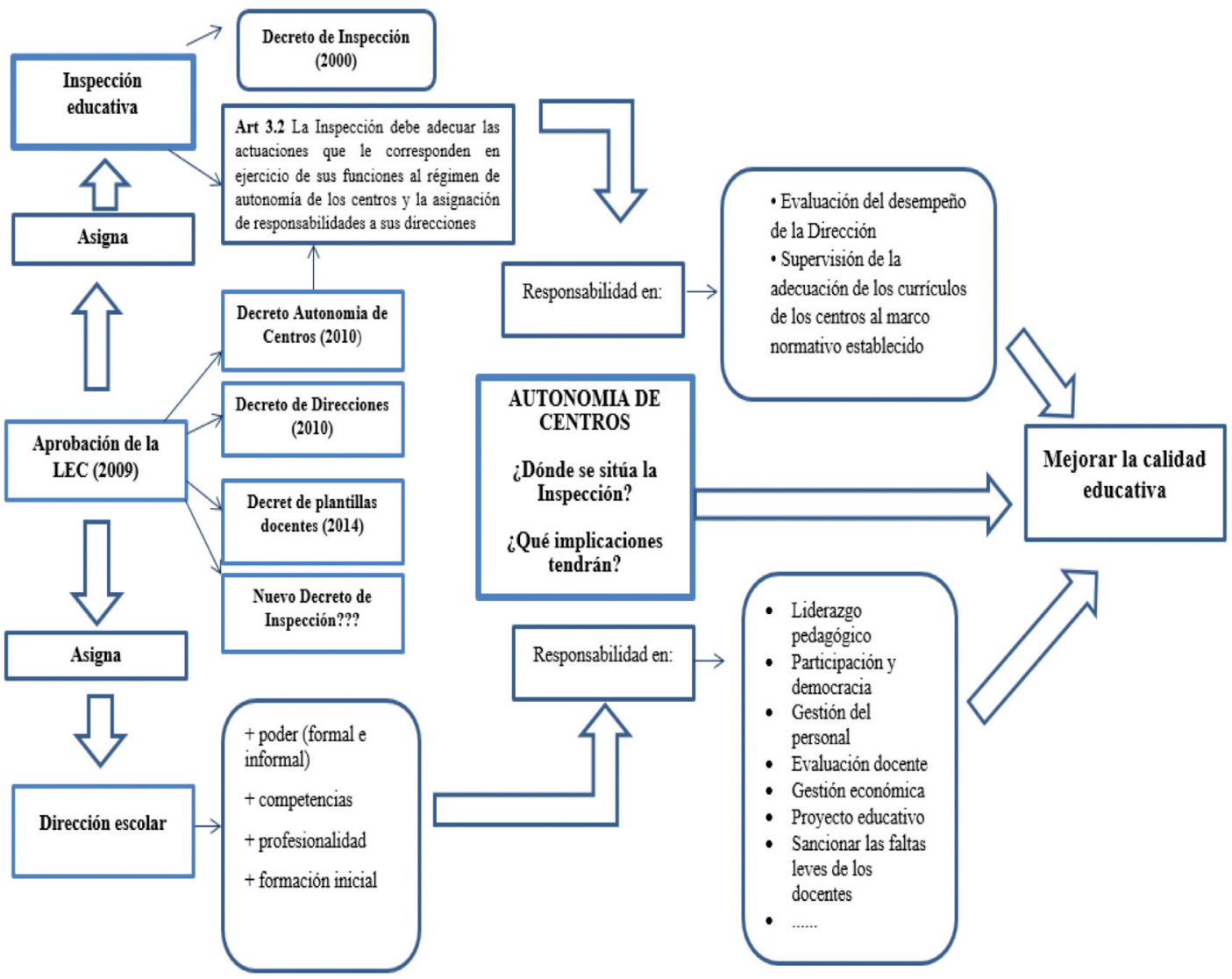

Nota. Elaboración propia.

Como se puede observar la finalidad principal seria la mejora de la calidad educativa, así también lo considera Moreno (2019) cuando afirma que la Inspección educativa, preserva y defiende la calidad de la educación, siendo esta un referente para la mejora del sistema educativo. Para Del Pozo (2016b), una de las atribuciones asignadas al director/a sería la supervisión de la práctica docente con la pérdida de exclusividad que tenía la Inspección educativa hasta ahora. Lo mismo sucede con la función de supervisar y evaluar el ejercicio de la función docente, colaborando directamente con la Inspección.

Siguiendo en esta misma línea, Colom (2011), considera que la Dirección queda muy reforzada, estableciéndose una serie de ámbitos de responsabilidad que crecen de forma significativa. No sucede lo mismo cuando nos referimos a la Inspección, se entiende que ésta deberá participar y colaborar, pero ahora desde una perspectiva de acompañamiento a cada centro, ya que, dependiendo de la autonomía de cada uno, las necesidades serán unas y no otras. Como propuesta para ubicar a la Inspección en el proceso de autonomía, Prat (2011) apuesta como actuación principal de la Inspección desarrollar los procesos de evaluación del sistema educativo y su participación en estos procesos. Esto conlleva enfocar la tarea de la Inspección, que no por ello pasa a ser menos importante, ya que suministra a las direcciones los inputs necesarios para desarrollar su labor.

\section{Método}

Siguiendo a Onwuegbuzie y Leech (2006), se plantea una investigación mixta con preponderancia cualitativa y el método del estudio de casos; más concretamente, el estudio colectivo de casos (Stake, 
2005), que implica el estudio de la Inspección educativa a partir de diferentes casos en situaciones diversas.

El objetivo general es: Analizar los cambios que se están produciendo en las funciones de la Inspección educativa en su relación e implicación con los centros educativos y las direcciones escolares a partir del desarrollo del Decreto de autonomía de Centros. Como objetivos específicos se plantean:

- Describir la situación actual de la Inspección educativa y la autonomía de centros en Cataluña

- Valorar la función inspectora en relación con el trabajo que desarrolla a partir del Decreto de Autonomía de centros.

- Analizar las percepciones de la Inspección educativa y la Dirección escolar en referencia a sus funciones y la relación entre ambos.

- Describir la aportación de la Inspección educativa en el proceso de la mejora de la calidad educativa en los centros que desarrollan la autonomía.

- Identificar propuestas de mejora para el ejercicio de la Inspección, dando respuesta a las necesidades que han surgido durante el estudio.

\section{Categorías de análisis y variables}

En la parte cualitativa del estudio se parte de la teoría Fundamentada: Método Comparativo Constante (Glaser y Straus, 1967). Este nos ayuda en la búsqueda de semejanzas y diferencias a través del análisis de los incidentes contenidos en los datos.

El hecho de categorizar, supone un proceso de agrupamiento. En este proceso de agrupación se crean las metacategorias entendidas éstas como un conjunto de categorías, según los criterios establecidos en la investigación y que permiten agrupar más información (Silva, 2008). Se parte de 18 categorías codificadas en temas y patrones, realizando una lectura de estas para encontrar las relaciones y similitudes entre ellas con la finalidad de realizar agrupamientos más reducidos. En esta revisión surgen 8 metacategorias de las 18 categorías que se definen en:

- Situación actual de la inspección educativa

- Situación actual de la autonomía de centros

- Relación Direcciones e Inspección Educativa

- Dualidad de funciones entre las direcciones escolares y la Inspección educativa

- Funciones de la inspección educativa en el proceso de autonomía de centros

- Aportaciones de la Inspección educativa en el marco de autonomía

- Necesidades de la inspección educativa en el marco autonómico

- Propuestas de mejora de la inspección educativa

Las técnicas y test estadísticos que se utilizan referentes a la parte cuantitativa de la investigación encontramos:

- En variables cualitativas (nominales): distribución de frecuencias y porcentajes.

- En variables cuantitativas: Descripción a las herramientas habituales de centralidad (media, mediana) y variabilidad (desviación estándar).

- Test U de Mann-Whitney y Test H de Kruskal-Wallis de diferencias para grupos independientes entre sí, en variables numéricas 
- Test Chi-cuadrado de dependencia entre variables categóricas.

- El nivel de significación fijado es el habitual 5\% (significativo si p <.05) análisis inferencial

También se utilizan estadísticas inferenciales con la finalidad de encontrar diferencias estadísticamente significativas entre las respuestas de los Inspectores/as. Se cruzan algunas de las variables dependientes generadas por las preguntas, con los dos factores seleccionados llamados (variables independientes) característicos de la muestra: 1) Situación profesional y 2) cargo en la Inspección.

Los métodos empleados son estado de tipo no-paramétrico, principalmente por el reducido $\mathrm{N}$ de encuestados; pero también por la dificultad de probar el ajuste a la normalidad de las variables Likert.

\section{Participantes}

La selección de la muestra varía dependiendo de la perspectiva cualitativa y cuantitativa y del instrumento elegido para la recogida de la información. Nuestro universo son los Inspectores e Inspectoras de educación. En cuanto a la población acota todos aquellos Inspectores/as que desarrollan sus tareas en Cataluña (243), que es el centro de estudio. Además, para la realización de las entrevistas en profundidad se ubican más concretamente en el territorio leridano. También se selecciona esta parte de territorio catalán por los grupos de discusión, donde también incorporamos a las Direcciones escolares para contrastar las percepciones de la Inspección educativa. Se comprende que los directores/as ofrecen la posibilidad de aportar visiones diferentes además de dar su respuesta para con la relación profesional entre Inspección educativa y Direcciones escolares.

\section{Instrumentos de recogida de información}

Para la elección de la instrumentalización (figura 2) que diera respuesta a los objetivos planteados se partieron de tópicos delimitados previamente por la revisión normativa y de la literatura existente, de acuerdo a autores como Hernández Sampieri, Fernández y Baptista (2010), Sandín (2003) y Taylor y Bogdan (2010). Las fases consideradas en el estudio y los instrumentos considerados se pueden concretar en:

Fase descriptiva:

- Análisis de documentos: Para la caracterización general de los inspectores se consultaron diferentes Decretos, Planes directores de la Inspección educativa, diferentes Leyes educativas, bases de datos, etc.

- Cuestionario: Constó de 28 preguntas de diferente tipología, divididas en 8 bloques teniendo presente los datos personales de identificación De un total de 243 inspectores, contestaron 88.

- Entrevista en Profundidad: Se realiza 12 entrevistas a Inspectores/as del Servicio Territorial de Enseñanza en Lleida con 15 preguntas divididas en 7 bloques de contenidos.

Fase interpretativa:

- Grupo de discusión: Se generan 2 grupos de discusión con los agentes educativos (inspección, Dirección escolar) para debatir sobre 5 preguntas iniciales abiertas a otras que fueron surgiendo. En primer grupo son 4 directores y 2 directoras de diferente tipología de centros; el segundo grupo está compuesto por 4 Inspectores y 1 Inspectora

Fase propositiva:

- Cuestionario: Intervinieron 6 expertos de la materia (agentes de la Administración educativa con mucha experiencia y con estrecha relación con la Inspección educativa), para contrastar los resultados obtenidos en la fase interpretativa. 
Figura 2

Fases estudio

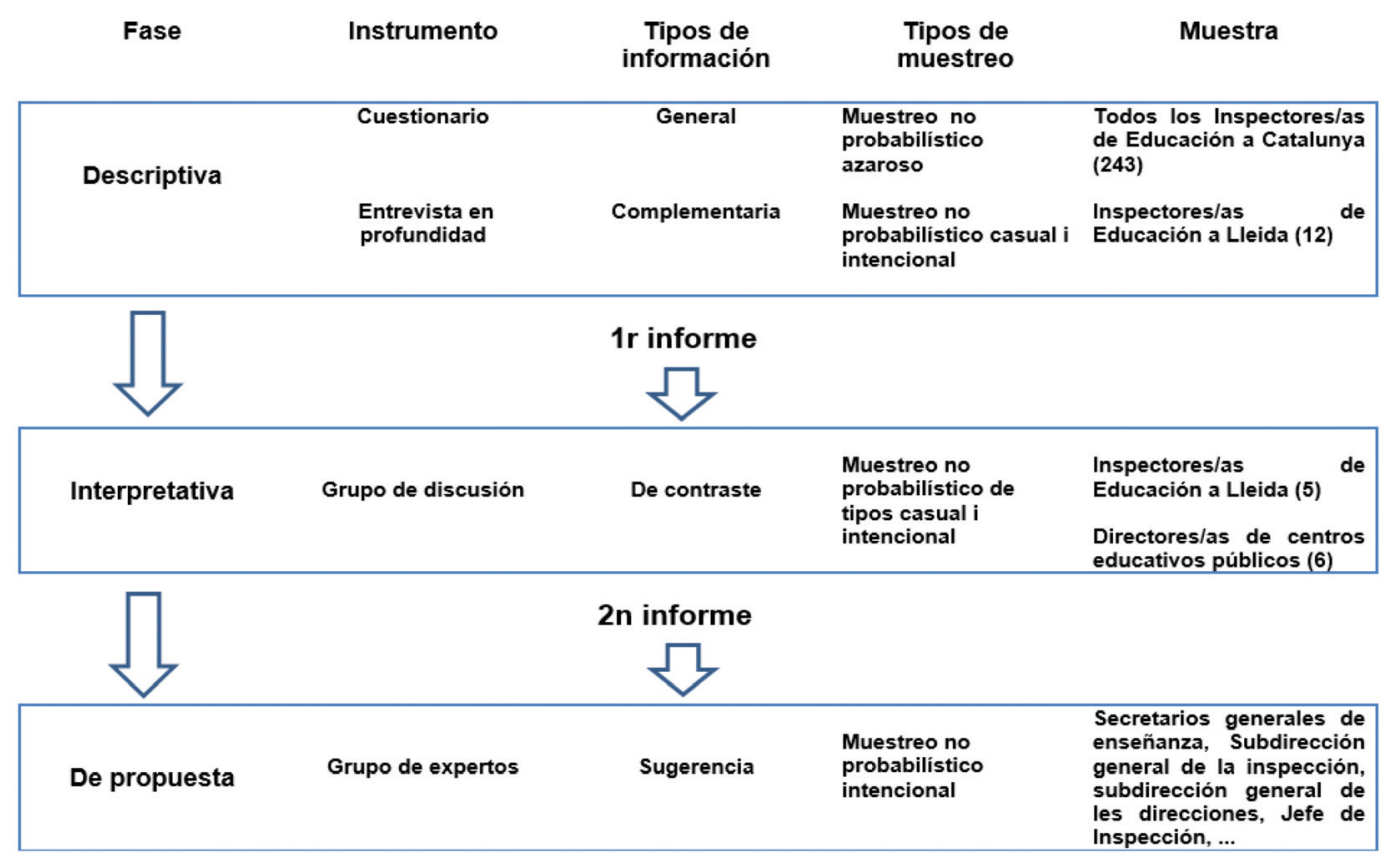

Nota. Elaboración propia.

\section{Análisis de datos}

Respecto al análisis de datos, el cuestionario se valida inicialmente por 12 especialistas de educación vinculados a la Inspección educativa y a las Direcciones escolares. La aplicación que se utiliza es Google Drive y la explotación de la información se realiza a través de la aplicación estadística IBM-SPSS-22. Para la estimación de la fiabilidad se utiliza la clásica ecuación alfa de Cronbach de consistencia interna, muy similar a la correlación intraclase, y que toma valores en el rango [0-1]. El resultado que se obtiene (783; IC al 95\%: 704-849) indica que la fiabilidad es alta y estadísticamente significativa $(\mathrm{p}<0,001)$.

El tratamiento estadístico del cuestionario contempla dos tipos de análisis: un descriptivo de las respuestas recogidas en el cuestionario, y un segundo inferencial. La muestra utilizada se consideró como estadísticamente representativa de la población de estudio, con un nivel de confianza del $95 \%$ y un margen de error del $\pm 8: 34 \%$, en el supuesto más desfavorable de varianza máxima $(P=Q=12,50)$.

Las entrevistas buscan la identificación y tratamiento a través de categorías, combinando procesos de revisión reiterada y de saturación de la documentación con la herramienta computacional ATLAS-TI (versión 7). Las meta-categorías resultantes fueron 8. La selección de los entrevistados fue, al principio, es casual o por accesibilidad; posteriormente, se realiza un muestreo intencional, seleccionando aquellos que cumplían con unos criterios previamente establecidos: paridad, situación laboral en la Inspección y tiempo trabajado. Cada entrevista y entrevistado tiene un código asignado (IE-01-Inspector/a de educación - entrevista número 01).

Para identificar validar y triangular información, se quiso crear diferentes grupos de discusión con los agentes educativos. El tipo de muestreo es igual que en la entrevista, casual a la hora de acceder a la población, pero intencional para seleccionarlos para el grupo de discusión. La selección de los grupos busca la diversidad siguiendo criterios como la experiencia, edad, sexo, etapa educativa, entre otros dependiendo del grupo.

En este caso también se codifica cada grupo de discusión:

- GDDCE-01. Grupo de discusión de directores/as de Centros educativos

- GDIE-01. Grupo de discusión de Inspectores/as de educación 
Las fases descriptiva e interpretativa se completan con una tercera fase propositiva que es sometida a validación a través de un cuestionario por 6 expertos del mundo educativo y la Inspección. Este grupo de expertos ayudan a contrastar los resultados obtenidos en la muestra seleccionada y a terminar de definir las propuestas de mejora surgidas de las conclusiones. El tipo de muestreo utilizado para la selección de la muestra fue totalmente intencional en este caso y vinculado a la identificación de personas especialistas (han escrito o participado en procesos normativos vinculados a la temática).

\section{Resultados}

Los resultados más significativos se presentan de manera sintética partiendo de las meta-categorías desarrolladas a partir del proceso de categorización y codificación de los datos obtenidos de los diferentes instrumentos para su triangulación.

\subsection{Situación actual de la autonomía de centros}

La autonomía de centros se considera necesaria para conseguir la mejora de la calidad educativa. Uno de los factores a tener en cuenta para su mejora, es la necesidad de tiempo para su implantación (cuadro 1).

Cuadro 1

Resultados pregunta 8 del cuestionario

\begin{tabular}{lcccccccc}
\hline & \multirow{2}{*}{} & \multicolumn{3}{c}{ \% del Grado de acuerdo } & & \multicolumn{2}{c}{ Descriptivos } \\
\cline { 3 - 8 } & & Nada & Poco & Bast. & Tot. & Media & Med. & Desv. Est. \\
\hline $\begin{array}{l}\text { 8. Necesidad de la } \\
\begin{array}{l}\text { autonomía de centros } \\
\text { para mejorar la calidad } \\
\text { educativa. }\end{array}\end{array}$ & 87 & 0,0 & 4,6 & 33,3 & 62,1 & 3,57 & 4,00 & 0,58 \\
\hline
\end{tabular}

Nota. Elaboración propia.

Yo pienso que sí es necesaria la autonomía de centros, ¿y por qué? las Direcciones del centro deben tener la suficiente autonomía para poder establecer sus objetivos, su progreso y sus procesos de trabajo, ... Pienso que si es necesario avanzar hacia la autonomía de centros. (EIE-08- 8: 1 (7: 7))

Se señala que la autonomía es buena para la mejora de la calidad educativa, aunque se percibe que no es efectiva. Además, la Inspección considera que las Direcciones no se la creen y les falta formación y decisión; y las Direcciones afirman que no tienen suficientes herramientas para ser autónomos y tomar las decisiones que comporta.

Se entiende que las malas praxis por parte de las direcciones escolares no deberían de producirse y, si sucede, la Inspección tiene herramientas para detectarlas y corregirlas.

\subsection{Situación actual de la inspección educativa}

Se apuesta por un liderazgo pedagógico de manera externa a través del asesoramiento a partir de los conocimientos adquiridos por la Inspección. Se considera de gran ayuda en la evolución y mejora pedagógica y de funcionamiento de un centro educativo. Las palabras que más se repiten es ayuda, asesoramiento y reparadores de problemas.

La Inspección debe conocerse, ser visible para todos y difundir sus funciones en el sistema educativo. Entre las dificultades operativas, encuentran que el porcentaje alto de comisiones de servicios $(74,5 \%)$, perjudica la estabilidad de la Inspección como Cuerpo (cuadro 2 ). 


\begin{tabular}{lccccc}
\hline & & \multicolumn{3}{c}{ Descriptivos } & \\
& $\mathrm{N}$ & Media & Med. & $\begin{array}{c}\text { Desv. } \\
\text { Est. }\end{array}$ & $\begin{array}{c}\text { Respuestas de mayor } \\
\text { frecuencia (\%) }\end{array}$ \\
\hline $\begin{array}{l}\text { 14. El 74,5\% de comisionados en la } \\
\begin{array}{l}\text { Inspección Educativa perjudica la } \\
\text { estabilidad de esta como Cuerpo. }\end{array}\end{array}$ & 86 & 2,76 & 3,00 & 0,98 & \\
\hline
\end{tabular}

Nota. Elaboración propia.

Para cerrar este apartado aportamos la valoración que hacen de su nivel de fortaleza como Institución, la nota numérica es de un aprobado alto cercano al notable.

Añadir, que aparte del análisis estadístico descriptivo en algunas preguntas del cuestionario, se realiza un segundo inferencial de las variables implicadas. Esto significa cruzar algunas de las variables dependientes generadas por las preguntas con los dos factores llamados (variables independientes) característicos de la muestra: 1) Situación profesional y 2) Cargo en la inspección.

Para los cruces del primer factor, se parte de dos niveles (Inspectores/as de educación funcionarios y Funcionarios docentes que ejercen como Inspectores /as) El segundo factor está definido con 3 niveles o categorías: Inspector /a jefe y adjunto/coordinadores /as / sin cargo actual. En cuanto al primer factor en función de la situación profesional, los resultados de los contrastes de las variables del cuestionario se presentan en el cuadro 3. Como se puede comprobar en ella, no se han encontrado diferencias significativas $(p>0,05)$ entre los Inspectores de Educación funcionarios y los funcionarios docentes que ejercen como Inspectores en las variables de la P.14 (Comisión de servicios perjudica...) y la P.15 (Fortaleza de la IE).

Cuadro 3

Análisis inferencial inspección educativa. Situación profesional

\begin{tabular}{|c|c|c|c|c|c|c|}
\hline & \multicolumn{2}{|c|}{ Funcionarios IE $(\mathrm{n}=27)$} & \multicolumn{2}{|c|}{ Doc. Inspect. $(\mathrm{n}=61)$} & \multicolumn{2}{|c|}{ Test de MW } \\
\hline & $\begin{array}{l}\text { Media } \\
\text { (D.E.) }\end{array}$ & Med. & $\begin{array}{l}\text { Media } \\
\text { (D.E.) }\end{array}$ & Med. & Valor & $\mathrm{p}$-valor \\
\hline $\begin{array}{l}\text { 14. El } 74,5 \% \text { de comisionados en la } \\
\text { Inspección Educativa perjudica la } \\
\text { estabilidad de la Inspección como } \\
\text { Cuerpo. }\end{array}$ & $\begin{array}{l}3,00 \\
(0,92)\end{array}$ & 3,00 & $\begin{array}{c}2,64 \\
(1,00)\end{array}$ & 3,00 & $1,64^{*}$ & 0,101 \\
\hline $\begin{array}{l}\text { 15. Valoración de la fortaleza de la } \\
\text { Inspección Educativa actualmente. }\end{array}$ & $\begin{array}{c}5,70 \\
(2,13)\end{array}$ & 6,00 & $\begin{array}{c}6,23 \\
(1,47)\end{array}$ & 6,00 & $0,95^{*}$ & 0,340 \\
\hline
\end{tabular}

Nota. ${ }^{*}$ No significativo $(\mathrm{p}>0,05)$. Elaboración propia.

El segundo factor en función del cargo que ejerce en la Inspección educativa, tampoco se han encontrado diferencias que puedan considerarse como estadísticamente significativas $(p>0,05)$ entre los cargos comparados en ninguna de las variables de esta sección (cuadro 4). 
Análisis inferencial inspección educativa. Cargo que se desempeña

\begin{tabular}{|c|c|c|c|c|c|c|c|c|}
\hline & \multicolumn{2}{|c|}{ Sin $\operatorname{cargo}(n=11)$} & \multicolumn{2}{|c|}{$\begin{array}{l}\text { Inspect. jefe y adj. } \\
\qquad(\mathrm{n}=36)\end{array}$} & \multicolumn{2}{|c|}{ Coord. $(n=41)$} & \multicolumn{2}{|c|}{ Test de KW } \\
\hline & $\begin{array}{l}\text { Media } \\
\text { (D.E.) }\end{array}$ & Med. & $\begin{array}{l}\text { Media } \\
\text { (D.E.) }\end{array}$ & Med. & $\begin{array}{l}\text { Media } \\
\text { (D.E.) }\end{array}$ & Med. & Valor & $\mathrm{p}$-valor \\
\hline $\begin{array}{l}\text { 14. El } 74,5 \% \text { de comisio- } \\
\text { nados en la Inspección } \\
\text { Educativa perjudica la } \\
\text { estabilidad de la Inspec- } \\
\text { ción como Cuerpo. }\end{array}$ & $\begin{array}{c}2,73 \\
(0,79)\end{array}$ & 3,00 & $\begin{array}{l}2,66 \\
(1,11)\end{array}$ & 3,00 & $\begin{array}{c}2,85 \\
(0,92)\end{array}$ & 3,00 & $0,57^{*}$ & 0,753 \\
\hline $\begin{array}{l}\text { 15. Valoración de la for- } \\
\text { taleza de la Inspección } \\
\text { Educativa actualmente. }\end{array}$ & $\begin{array}{c}5,91 \\
(1,04)\end{array}$ & 6,00 & $\begin{array}{c}6,11 \\
(2,06)\end{array}$ & 7,00 & $\begin{array}{c}6,07 \\
(1,54)\end{array}$ & 6,00 & $0,71^{*}$ & 0,701 \\
\hline
\end{tabular}

Nota. * No significativo $(\mathrm{p}>0,05)$. Elaboración propia.

La vertiente cualitativa también refuerza estos datos e, incluso, encontramos opiniones que se quejan de esta posición laboral y lo relacionan con la independencia como Cuerpo de Inspección.

Me he encontrado con alguna Inspectora que cuando he tenido algún problema y, para no mojarse, ha tenido problemas. No ha velado por resolver el problema en el centro por miedo a su situación laboral. (GD1 DCE - 01:30 (43:43))

\subsection{Funciones de la inspección educativa en el proceso de autonomía de centros}

Los participantes consideran que las funciones actuales de la Inspección no se actualizan al nuevo marco educativo y tampoco están ubicadas en el nuevo marco de autonomía (cuadro 5).

Cuadro 5

Resultados pregunta 19.1 del cuestionario

\begin{tabular}{llccccccc}
\hline & & \multicolumn{3}{c}{ \% Grado de acuerdo } & & Descriptivos \\
\cline { 3 - 7 } & $\mathrm{N}$ & Nada & Poco & Bast. & Tot. & Media & $\begin{array}{c}\text { Med. } \\
\text { Desv. } \\
\text { Est. }\end{array}$ \\
\hline $\begin{array}{l}\text { 19.1. Las funciones ac- } \\
\begin{array}{l}\text { tuales de la Inspección } \\
\text { no se actualizan al nuevo } \\
\text { marco educativo. }\end{array}\end{array}$ & 86 & 7,0 & 40,7 & 45,3 & 7,0 & 2,52 & 3,00 & 0,73 \\
\hline
\end{tabular}

Nota. Elaboración propia.

Señalan que asesorar, evaluar y supervisar son las funciones más significativas y donde se invierte mayor tiempo de trabajo. De las tres funciones, la que más dedicación ocupa es la evaluación, aunque muchas veces se trabaja de manera transversal. Ubicados en la autonomía de centros, el asesoramiento es una de las funciones más importantes a desarrollar. La visión exterior de la Inspección, el conocimiento del trabajo que se hace en muchos centros o la experiencia de muchos años son factores a tener en cuenta. Un buen ejemplo sería el que aporta el participante 7:

La Inspección siempre influye por la vía del asesoramiento que, está, en definitiva, en dar una opinión. Es difícil establecer diferencias entre supervisión, evaluación y asesoramiento. Cuando vas a un centro, normalmente vas a supervisar un elemento, y puede surgir la necesidad de hacer un asesoramiento o bien una evaluación formativa. Los inspectores cuando van a un centro van con el triángulo de nuestra función: supervisión, evaluación y asesoramiento. (EIE-07- 7: 9 (38:38)) 


\subsection{Relación direcciones e inspección educativa}

En referencia al Decreto de Direcciones escolares (2010), consideran que las funciones, tareas y el rol de los directores y directoras han supuesto cambios. Aspectos como el ser considerados autoridad pública, tener mayor poder de decisión en el currículo, metodología, plantillas o mayor responsabilidad en los resultados de los centros educativos son algunas de las novedades (cuadro 6).

Destacan la importancia de la formación para liderar un centro educativo por parte de las direcciones. El paso de docente a director/a es muy rápido y no hay la suficiente formación para adaptarse a este nuevo rol. Necesidades formativas en liderazgo, en toma de decisiones, en gestionar el personal, evaluar o entrar en el aula son algunas de las carencias cuestionadas desde la visión de la Inspección.

Cuadro 6

Resultados pregunta 20.2 y 20.3. del cuestionario

\begin{tabular}{|c|c|c|c|c|c|c|c|c|}
\hline & \multirow[b]{2}{*}{$\mathrm{N}$} & \multicolumn{4}{|c|}{ \% Grado de acuerdo } & \multicolumn{3}{|c|}{ Descriptivos } \\
\hline & & Nada & Poco & Bast. & Tot. & Media & Med. & $\begin{array}{c}\text { Desv. } \\
\text { Est. }\end{array}$ \\
\hline $\begin{array}{l}\text { 20.2. El objetivo de mejo- } \\
\text { ra de la calidad del centro } \\
\text { requiere del trabajo con- } \\
\text { junto que se produzcan } \\
\text { entre estos dos agentes. }\end{array}$ & 88 & 0,0 & 4,5 & 40,9 & 54,5 & 3,50 & 4,00 & 0,59 \\
\hline $\begin{array}{l}\text { 20.3. Los diferentes de- } \\
\text { cretos han supuesto cam- } \\
\text { bios en la relación entre } \\
\text { la Inspección, los centros } \\
\text { docentes y las direcciones } \\
\text { escolares. }\end{array}$ & 88 & 2,3 & 23,9 & 50,0 & 23,9 & 2,95 & 3,00 & 0,76 \\
\hline
\end{tabular}

Nota. Elaboración propia.

La Inspección considera, en este contexto, que puede ayudar a formar mejores líderes de los centros educativos, pero también a regular si el liderazgo que se está desarrollando en el centro es correcto (cuadro 7).

Cuadro 7

Resultados pregunta 21 del cuestionario

\begin{tabular}{|c|c|c|c|c|c|c|c|c|}
\hline & \multirow[b]{2}{*}{$\mathrm{N}$} & \multicolumn{4}{|c|}{$\%$ Grado de acuerdo } & \multicolumn{3}{|c|}{ Descriptivos } \\
\hline & & Nada & Poco & Bast. & Tot. & Media & Med. & $\begin{array}{c}\text { Desv. } \\
\text { Est. }\end{array}$ \\
\hline $\begin{array}{l}\text { 21. La Inspección, dentro } \\
\text { de sus funciones, está } \\
\text { ayudando a mejorar el } \\
\text { liderazgo de las Direccio- } \\
\text { nes ante las dificultades. }\end{array}$ & 88 & 0,0 & 3,4 & 55,7 & 40,9 & 3,38 & 3,00 & 0,55 \\
\hline
\end{tabular}

Nota. Elaboración propia.

\subsection{Dualidad de funciones entre las Direcciones escolares y la Inspección educativa}

La Inspección educativa indica que no le supone ningún problema este aumento de funciones a las Direcciones escolares. Lo viven de una manera positiva y entienden que el modelo debe ser este y no identifican duplicidad de funciones. Se entiende que donde no llegue la Dirección llegará la Inspección, por lo tanto, se complementan con un mismo objetivo. 
La Dirección tiene autonomía y debe ejercerla y la Inspección asesora, supervisa y evalúa, ninguna duplicidad de funciones, como señala uno de los participantes:

El aumento de autonomía de centros no implica una pérdida de las tareas del inspector. Todo el tema de supervisión y asesoramiento continúa, y cada vez más los centros te lo piden. No creo que suponga ninguna pérdida de nada; es más, considero que es mucho mejor el modelo este, donde los Directores tengan más autoridad con el Claustro que el modelo anterior. (EIE-04- 4: 6 (25:25))

\subsection{Aportaciones de la Inspección educativa en el marco de autonomía}

Sobre las aportaciones de la Inspección educativa al sistema educativo se señalan: la evaluación centros, la supervisión de adjudicaciones de plazas, la arbitrariedad y propuestas de mejora en los centros después de analizar los resultados de los centros o el análisis de resultado (cuadro 8).

Cuadro 8

Resultados pregunta 23 del cuestionario

\begin{tabular}{|c|c|c|c|c|c|c|c|c|c|}
\hline & \multirow[b]{2}{*}{$\mathrm{N}$} & \multirow[b]{2}{*}{ Nada } & \multicolumn{3}{|c|}{$\%$ de respuesta } & \multirow[b]{2}{*}{ Muc. } & \multicolumn{3}{|c|}{ Descriptivos } \\
\hline & & & Poco & Norm. & Bast. & & Media & Med. & $\begin{array}{c}\text { Desv. } \\
\text { Est. }\end{array}$ \\
\hline $\begin{array}{l}\text { 23.10. En la avaluación } \\
\text { de centro función docen- } \\
\text { te y directiva, planes y } \\
\text { programas ... }\end{array}$ & 88 & 0,0 & 0,0 & 5,7 & 23,9 & 70,5 & 4,65 & 5,00 & 0,59 \\
\hline $\begin{array}{l}\text { 23.12. En la solución de } \\
\text { quejas y denuncias. }\end{array}$ & 88 & 0,0 & 0,0 & 1,1 & 34,1 & 64,8 & 4,64 & 5,00 & 0,51 \\
\hline $\begin{array}{l}\text { 23.9. En el asesoramien- } \\
\text { to a los centros, equipo } \\
\text { directivo, docentes. }\end{array}$ & 86 & 0,0 & 0,0 & 4,7 & 34,9 & 69,5 & 4,56 & 5,00 & 0,59 \\
\hline $\begin{array}{l}\text { 23.1. En las direcciones } \\
\text { escolares }\end{array}$ & 88 & 0,0 & 0,0 & 4,5 & 51,1 & 44,3 & 4,40 & 4,00 & 0,58 \\
\hline $\begin{array}{l}\text { 23.6. En la resolución de } \\
\text { conflictos }\end{array}$ & 88 & 2,3 & 0,0 & 11,4 & 51,1 & 35,2 & 4,17 & 4,00 & 0,80 \\
\hline $\begin{array}{l}\text { 23.8. En la orientación a } \\
\text { los centros. }\end{array}$ & 88 & 2,3 & 0.0 & 11,4 & 54,5 & 31,8 & 4,14 & 4,00 & 0,79 \\
\hline $\begin{array}{l}\text { 23.3. En la mejora de la } \\
\text { calidad educativa }\end{array}$ & 87 & 2,3 & 0,0 & 11,5 & 69,0 & 17,2 & 3,99 & 4,00 & 0,71 \\
\hline $\begin{array}{l}\text { 23.13. En la difusión de } \\
\text { buenas prácticas. }\end{array}$ & 88 & 8,0 & 0,0 & 14,8 & 61,4 & 15,9 & 3,77 & 4,00 & 0,99 \\
\hline 23.5. En la mediación & 88 & 4,5 & 0,0 & 26,1 & 53,4 & 15,9 & 3,76 & 4,00 & 0,88 \\
\hline $\begin{array}{l}\text { 23.11. En el trabajo en } \\
\text { red. }\end{array}$ & 88 & 6,8 & 0,0 & 29,5 & 47,7 & 15,9 & 3,66 & 4,00 & 0,98 \\
\hline $\begin{array}{l}\text { 23.4. En la formación del } \\
\text { profesorado y equipos } \\
\text { directivos. }\end{array}$ & 88 & 14,8 & 0,0 & 22,7 & 51,1 & 11,4 & 3,44 & 4,00 & 1,17 \\
\hline $\begin{array}{l}\text { 23.7. En la innovación } \\
\text { educativa }\end{array}$ & 88 & 9,1 & 0,0 & 35,2 & 48,9 & 6,8 & 3,44 & 4,00 & 0,97 \\
\hline 23.2. Al profesorado & 88 & 13,6 & 0,0 & 42,0 & 37,5 & 6,8 & 3,24 & 3,00 & 1,07 \\
\hline
\end{tabular}

Nota. Elaboración propia.

\subsection{Necesidades de la inspección educativa en el marco autonómico}

El cuadro 9 recoge las necesidades de la Inspección educativa en el marco autonómico de manera general y específica. Están ordenados de más a menos grado de acuerdo, pudiendo observar una concentración 
en los dos valores más altos (bastante y total acuerdo) y bastante homogeneidad en las respuestas (con desviaciones estándar bajas).

Cuadro 9

Resultados pregunta 25 del cuestionario

\begin{tabular}{|c|c|c|c|c|c|c|c|c|}
\hline & \multirow[b]{2}{*}{$\mathrm{N}$} & \multicolumn{4}{|c|}{$\%$ Grado de acuerdo } & \multicolumn{3}{|c|}{ Descriptivos } \\
\hline & & Nada & Poco & Bast. & Tot. & Media & Med. & $\begin{array}{c}\text { Desv. } \\
\text { Est. }\end{array}$ \\
\hline $\begin{array}{l}\text { 25.9. Sintetizar la multi- } \\
\text { tud de funciones y tareas } \\
\text { que desarrolla la Inspec- } \\
\text { ción, ... }\end{array}$ & 87 & 0,0 & 10,3 & 41,4 & 48,3 & 3,38 & 3,00 & 0,67 \\
\hline $\begin{array}{l}\text { 25.6. Formación inicial } \\
\text { común reglada para los } \\
\text { Inspectores que se incor- } \\
\text { poran al Cuerpo }\end{array}$ & 88 & 0,0 & 9,1 & 44,3 & 46,6 & 3,38 & 3,00 & 0,65 \\
\hline $\begin{array}{l}\text { 25.4. Modificar la ordena- } \\
\text { ción actual de la inspec- } \\
\text { ción para adaptarse a los } \\
\text { retos educativos actuales }\end{array}$ & 87 & 0,0 & 9,2 & 46,0 & 44,8 & 3,36 & 3,00 & 0,65 \\
\hline $\begin{array}{l}\text { 25.2. Cambiar el modelo } \\
\text { de control burocrático } \\
\text { por un menos burocrático }\end{array}$ & 88 & 1,1 & 5,7 & 55,7 & 37,5 & 3,30 & 3,00 & 0,63 \\
\hline $\begin{array}{l}\text { 25.1. Aumentar el grado } \\
\text { de independencia como } \\
\text { Cuerpo de Inspección }\end{array}$ & 88 & 0,0 & 11,4 & 50,0 & 38,6 & 3,27 & 3,00 & 0,66 \\
\hline $\begin{array}{l}\text { 25.5. Formación especí- } \\
\text { fica desde la aprobación } \\
\text { del Decreto de autonomía } \\
\text { para implicarse en todo } \\
\text { este proceso }\end{array}$ & 88 & 0,0 & 13,6 & 51,1 & 35,2 & 3,22 & 3,00 & 0,57 \\
\hline $\begin{array}{l}\text { 25.8. Recursos tecno- } \\
\text { lógicos para facilitar el } \\
\text { desarrollo de la función } \\
\text { inspectora }\end{array}$ & 87 & 0,0 & 1,6 & 52,9 & 34,5 & 3,22 & 3,00 & 0,66 \\
\hline $\begin{array}{l}\text { 25.7. Incrementar el } \\
\text { número de inspectores } \\
\text { en Cataluña para ofrecer } \\
\text { una mayor calidad del } \\
\text { servicio en los centros }\end{array}$ & 88 & 6,8 & 13,6 & 47,7 & 31,8 & 3,05 & 3,00 & 0,86 \\
\hline
\end{tabular}

Nota. Elaboración propia.

\subsection{Propuestas de mejora de la inspección educativa}

Hay propuestas de mejora muy diversas y sobre diferentes aspectos como revisar el acceso a la Inspección educativa, su organización, el papel que debe tener en Innovación o qué tipo de formación requiere entre otros (tabla 10, ordenados los ítems de mayor a menor grado de acuerdo). El mayor acuerdo se refiere a la realización de un nuevo Decreto de la Inspección (seguido muy de cerca de la propuesta para mejorar la estabilidad del cuerpo de inspección). 


\begin{tabular}{|c|c|c|c|c|c|c|c|c|}
\hline & \multirow[b]{2}{*}{$\mathrm{N}$} & \multirow[b]{2}{*}{ Nada } & \multicolumn{3}{|c|}{ \% Grado de acuerdo } & \multicolumn{3}{|c|}{ Descriptivos } \\
\hline & & & Poc. & Bast. & Tot. & Media. & Med. & $\begin{array}{c}\text { Desv. } \\
\text { Est. }\end{array}$ \\
\hline $\begin{array}{l}\text { 27.2. Actualizar un nuevo } \\
\text { Decreto de Inspección }\end{array}$ & 87 & 0,0 & 5,7 & 42,5 & 51,7 & 3,46 & 4,00 & 0,61 \\
\hline $\begin{array}{l}\text { 27.14. Mejorar la esta- } \\
\text { bilidad del Cuerpo de } \\
\text { Inspección }\end{array}$ & 86 & 0,0 & 5,8 & 43,0 & 51,2 & 3,45 & 4,00 & 0,61 \\
\hline $\begin{array}{l}\text { 27.11. Trabajar por una } \\
\text { independencia de la Ins- } \\
\text { pección educativa }\end{array}$ & 88 & 0,0 & 14,8 & 37,5 & 47,7 & 3,33 & 3,00 & 0,72 \\
\hline $\begin{array}{l}\text { 27.8. Aumentar la partici- } \\
\text { pación de la Inspección en } \\
\text { la innovación educativa. }\end{array}$ & 88 & 0,0 & 9,1 & 56,8 & 34,1 & 3,25 & 3,00 & 0,61 \\
\hline $\begin{array}{l}\text { 27.3. Redefinir las fun- } \\
\text { ciones de la Inspección } \\
\text { educativa. }\end{array}$ & 87 & 1,1 & 11,5 & 51,7 & 35,6 & 3,22 & 3,00 & 0,69 \\
\hline $\begin{array}{l}\text { 27.4. Mejorar la formación } \\
\text { por una mejor especiali- } \\
\text { zación en las etapas edu- } \\
\text { cativas i en las materias } \\
\text { curriculares }\end{array}$ & 87 & 1,1 & 11,5 & 52,9 & 34,5 & 3,21 & 3,00 & 0,68 \\
\hline $\begin{array}{l}\text { 27.9. Dirigir la Inspección } \\
\text { a un Cuerpo menos buro- } \\
\text { crático }\end{array}$ & 88 & 1,1 & 14,8 & 48,9 & 35,2 & 3,18 & 3,00 & 0,72 \\
\hline $\begin{array}{l}27.15 \text {. Dotar de mayores } \\
\text { capacidades estratégicas } \\
\text { y recursos tecnológicos } \\
\text { para facilitar la función } \\
\text { inspectora }\end{array}$ & 87 & 0,0 & 13,8 & 55,2 & 31,0 & 3,17 & 3,00 & 0,65 \\
\hline $\begin{array}{l}\text { 27.1. Crear un nuevo mo- } \\
\text { delo de ordenación de la } \\
\text { Inspección }\end{array}$ & 86 & 1,2 & 16,3 & 47,7 & 34,9 & 3,16 & 3,00 & 0,73 \\
\hline $\begin{array}{l}\text { 27.10. Ganar visibilidad } \\
\text { a través de la visita a los } \\
\text { centros educativos. }\end{array}$ & 85 & 2,4 & 15,3 & 45,9 & 36,5 & 3,16 & 3,00 & 0,77 \\
\hline $\begin{array}{l}\text { 27.5. Crear un progra- } \\
\text { ma de formación inicial } \\
\text { común para todos los } \\
\text { inspectores de educación, } \\
\text { equiparable a un postgra- } \\
\text { do o máster universitario. }\end{array}$ & 88 & 3,4 & 20,5 & 37,5 & 38,6 & 3,11 & 3,00 & 0,85 \\
\hline $\begin{array}{l}\text { 27.13. Fortalecer la figura } \\
\text { de la Inspección en re- } \\
\text { ferencia a la comunidad } \\
\text { educativa }\end{array}$ & 88 & 1,1 & 14,8 & 58,0 & 26,1 & 3,09 & 3,00 & 0.67 \\
\hline $\begin{array}{l}\text { 27.6. Aumentar los recur- } \\
\text { sos humanos (inspecto- } \\
\text { res) para poder ofrecer } \\
\text { mayor calidad del servicio } \\
\text { a los centros }\end{array}$ & 88 & 4,5 & 20,5 & 43,2 & 31,8 & 3,02 & 3,00 & 0,84 \\
\hline $\begin{array}{l}\text { 27.7. Revisar la estructura } \\
\text { organizativa de la Inspec- } \\
\text { ción }\end{array}$ & 88 & 1,1 & 26,1 & 43,2 & 29,5 & 3,01 & 3,00 & 0,78 \\
\hline 27.12. Redefinir su misión. & 87 & 1,1 & 23,0 & 52,9 & 23,0 & 2,98 & 3,00 & 0,72 \\
\hline
\end{tabular}

Nota. Elaboración propia. 


\section{Conclusiones y discusión}

Se presentan de acuerdo con los objetivos planteados y se acompañan de comentarios relacionados con la interpretación de los resultados.

\section{Objetivo 1: Conocer la situación actual de la Inspección educativa y la autonomía de centros en Cataluña}

En referencia a la autonomía de centros, se mantienen dudas y su realidad no es nada fácil de desarrollar, dependiendo de las personas para su buen funcionamiento y sin obviar que pueden surgir problemas. Pero, en general, se piensa que la autonomía ayuda a situar el centro educativo en el foco de la mejora del sistema educativo y como un factor importantísimo del éxito escolar. Todos los informantes están de acuerdo en que la autonomía es buena, aunque no es efectiva, habiendo desacuerdo son las causas: la Inspección considera que las Direcciones no la creen y les falta formación y decisión; y las Direcciones afirman que no tienen suficientes herramientas para ser autónomos y tomar estas decisiones.

Muchos son los autores (Bolívar, 1994; Estruch, 2006, 2009; Graells, 2010b; Larruzea, 2011; Marchesi, 2004; Torres Rubio, 2010) que en sus estudios sobre la autonomía de centros también se habían cuestionados dudas. Gairín (2005), por ejemplo, se plantea algunas cuestiones como: ¿Cómo compaginar los procesos de legitimación con los de solidaridad? ¿Qué mecanismos de control explícitos o implícitos se establecerán? ¿Cómo superar las contradicciones a nivel político y social entre el discurso de autonomía y una práctica muchas veces insolidaria? ¿Se respetarán las decisiones que los centros adopten en el marco de la autonomía?

Pero, más allá de las dudas e inconvenientes, se percibe desde propia Inspección educativa la necesidad de implantar la autonomía de centros en el sistema educativo actual, para mejorar la calidad educativa. Así, también lo piensan Graells y García (2010) cuando afirman que el Decreto de autonomía dotaría a los centros de los instrumentos necesarios para desarrollar proyectos educativos que tengan incidencia real en la calidad educativa.

En cuanto a la situación de la Inspección educativa, la buena implementación de la autonomía, requiere de una Inspección que acompañe a los centros educativos a través del asesoramiento, la evaluación y la supervisión. Se busca un inspector menos burocrático y controlador, y, a cambio, se propone que sea más asesor, más innovador, más formativo y con mayor visibilidad en el centro. Estas funciones asignadas ayudarían a mantener la equidad deseada en la educación.

No se entendería el sistema educativo catalán sin la figura y el trabajo diario de la Inspección. Asimismo, piensa Larruzea (2011, p.124), cuando afirma que: "La Inspección es una pieza clave en la implantación de la autonomía. Se necesita una Inspección que no esté entrampada en gestiones burocráticas y que refuerce su perfil asesor hacia los centros, repensando sus funciones. Es una pieza fundamental en la relación Administración - Centros".

Objetivo 2. Valorar la función inspectora en relación al trabajo que desarrollan a partir del Decreto de Autonomía de centros.

Para la Inspección educativa, las funciones no han cambiado, se han adaptado y ello implica muchas veces invertir más dedicación en unas que en otras. Además, la opinión más común es que estas funciones que rigen la Inspección no hay que cambiarlas, únicamente ajustarlas.

Estos resultados coinciden con las palabras de Secadura (2013), cuando considera que la Inspección educativa sigue amarrada a criterios organizativos y funcionales que nacieron con el desarrollo de Ley General de la Educación de 1970. Normalmente los inspectores continúan haciendo su trabajo en una zona educativa exclusiva, durante un tiempo determinado, donde se les asigna un grupo de centros y con respecto a los que mantienen una supervisión individualista, y donde el enfoque hacia un seguimiento cotidiano de rutinas burocráticas es de poco calado.

Ante ese enfoque, se cree que la Inspección necesita de otros compromisos y estructuras, con un ámbito funcional que responda a las necesidades socioeducativas reales. Se propone, al respecto, que las Administraciones públicas realicen todo un proceso de cambio relevante en todo el contexto, aprovechando la valía de los propios profesionales. 
Pérez (2011) afirma que aún es pronto para hacer valoraciones y propuestas: "faltan todavía muchas decisiones para vislumbrar tendencias al respecto, pero lo que parece evidente es que está cambiando el escenario. Lo menos inteligente sería ignorarlo y cerrar los ojos” (pp. 3-4).

Respecto a las dificultades para el ejercicio de la inspección, las más repetidas son la multitud de funciones y tareas en sus actuaciones. Por ello, no es de extrañar la reclamación de una mayor definición de funciones y orientación hacia una especialización que ayude a desarrollar el talento de cada Inspector/a

Las funciones básicas de asesoramiento, evaluación y supervisión no han cambiado con la autonomía, concordando con aquellos países de Europa donde también se desarrolla la autonomía, tal y como afirma Vera (2014). Es cierto que, con el incremento de autonomía de centro, estas funciones han modificado su repercusión, y la que más afectación ha recibido ha sido la de control: mayor participación y funciones a la comunidad educativa, le ha hecho perder importancia. Por otra parte, la evaluación, la rendición de cuentas y el asesoramiento, han ganado en notoriedad, siendo ejes principales en todo el proceso de autonomía.

Como parte crítica, las Direcciones escolares perciben que la Inspección educativa tiene cierta preocupación por los resultados educativos, olvidándose, muchas veces, de ayudar en el proceso. Se quejan de que no escuchan ideas, proyectos, innovación sino sólo se habla de resultados. Por lo tanto, agradecerían más asesoramiento y no tanta evaluación ligada a supervisión.

Objetivo 3. Analizar las percepciones de la Inspección educativa y la Dirección escolar en referencia a sus funciones y la relación entre ambos.

La relación que se forme entre Dirección e Inspección determinará las implicaciones de cada uno en el proceso de autonomía. El objetivo de cambio educativo y de mejora de la calidad del centro, dependerá mucho del trabajo conjunto que se produzca entre estos dos agentes.

La Inspección educativa no ve problemas en el acercamiento a la dirección ni en compartir competencias que ante tenía en exclusiva. Prat (2011), analiza esta misma situación cuando afirma que:

Autonomía y asignación de responsabilidades de las Direcciones son ahora los límites claros y definidos en los que se deberá mover la Inspección en sus atribuciones. El problema radica en encontrar los mecanismos y las complicidades necesarias para que cada uno haga el trabajo que le corresponde, no se den duplicidades de funciones y el sistema educativo quede fortalecido contribuyendo a la mejora de la educación. (p. 20)

Artigal y Rios (2011), no ven tampoco motivos de conflictividad entre los dos agentes. Entienden que la Inspección puede ser muy válida para ayudar a detectar los puntos fuertes y débiles y también las oportunidades de mejora y las iniciativas de innovación. Sin entrar en las funciones de cada uno, sí que consideran que hay una serie de acciones donde pueden colaborar apoyando un proyecto y un liderazgo compartido. Estas actuaciones, que se complementan, ayudarían a construir hojas de ruta y planes de mejora.

Los participantes de la Inspección educativa y de las Direcciones escolares consideran que el asesoramiento ayuda a fortalecer esta relación. Sin embargo, en las otras funciones como son la evaluación y la supervisión de las Direcciones, estos últimos consideran que limita la relación mencionada. El aspecto que menos ayuda a esta relación sería la supervisión y aprobación de la continuidad en el cargo de la Dirección escolar. Es un hecho que no pasa muy a menudo, pero no siempre ayuda a generar buena relación en este binomio profesional.

La Inspección educativa percibe que puede ayudar mucho en que el liderazgo directivo mejore a través del asesoramiento, la evaluación y la supervisión. Afirman que pueden ayudar a formar mejores líderes en los centros educativos, pero también a regular si el liderazgo que se está desarrollando en el centro es correcto.

Objetivo 4. Conocer la aportación de la Inspección educativa en el proceso de la mejora de la calidad educativa en los centros que desarrollan la autonomía.

Entre las aportaciones más comentadas de forma abierta a través de las entrevistas y grupo de discusión que ha expresado la Inspección educativa, cabe citar: Análisis de resultados, Contrastar entornos de aprendizaje pedagógicos, Apoyo a los proyectos de centros, Sugerencia de propuestas de mejora en el centro y Supervisión. 
Estas aportaciones van en la línea que sugiere García Andreu (2013), cuando afirma que la Inspección educativa debe reconocer la vida real de los centros y de las aulas y no circunscribirse a meras tareas de burocracia. Para esta meta sugiere que las actuaciones de la Inspección educativa cooperen y refuercen la formación del profesorado, la formación específica previa de los equipos directivos y la puesta en marcha de las evaluaciones diagnósticas.

La valoración de la Inspección es muy positiva sobre aportaciones en todo el proceso de autonomía de centros. Consideran que son pieza importante en todas las acciones que desarrollan, ayudando al despliegue de la autonomía y dando apoyo a las Direcciones escolares con su experiencia, a mejorar la calidad educativa de sus centros educativos. Son influyentes y ayudan en aspectos tan importantes como la evaluación de los centros y la evaluación de los directores, en la formación, en el análisis de resultados y en sus pertinentes propuestas de mejora, entre otros. Y todavía queda camino por recorrer en atención al profesorado, innovación educativa, formación del profesorado y equipos directivos y el trabajo en red.

\section{Objetivo 5. Identificar propuestas de mejora para el ejercicio de la Inspección, dando respuesta a las necesi- dades de la Inspección educativa.}

Diferentes autores se centran en aspectos mejorables y que pueden ayudar a mejorar como Inspección. Jiménez (2016) visualiza una nueva Inspección "profesionalizada, menos burocrática y con una imagen más prestigiosa en el mundo educativa general” (p.61). En cambio, Castán (2016), pide mayor contacto con el profesorado, basándose en que los estudios internacionales coinciden con que los profesores son el principal valor de un sistema educativo.

Estañán (2014) afirma que la burocracia administrativa consume buena parte de los tiempos de la Inspección junto con su participación y colaboración en los procesos de evaluación externa. Por esta razón, considera un reto la participación de los miembros de la Inspección educativa en organizaciones y asociaciones que promuevan la reflexión y la innovación pedagógico. También Silva (2008) apuesta por mejorar la Innovación a través de la figura de la Inspección. Lo argumenta afirmando que, si alguien tiene la oportunidad de acompañar durante el camino a un determinado número de centros, esta persona es el inspector. Su ubicación dentro del sistema posibilita un acercamiento y unas condiciones propicias para lograr este proceso de innovación.

Los resultados ofrecidos en el estudio van muy en consonancia con los autores mencionados y otros como los de Camacho (2015), Galicia (2016) o Silva (2013). Actualizar un nuevo Decreto de la Inspección sería la propuesta más reivindicada, seguido de otros como mejorar la estabilidad del cuerpo de Inspección, independencia de la Inspección educativa, aumentar la participación de la Inspección en la innovación educativa, mejorar la formación para una mejor especialización en las etapas educativas y en las materias curriculares, dirigir la Inspección a un cuerpo menos burocrático, dotar de mayores capacidades estratégicas y recursos tecnológicos para facilitar la función inspectora, crear un nuevo modelo de ordenación de la Inspección, ganar visibilidad a través de la visita a los centros educativos o redefinir su misión.

Visibilizarse, reivindicar, trabajar en equipo, acompañar, ir todos a una, serían algunos de los ingredientes que recomienda la fórmula para mejorar la Inspección educativa. Si no se sigue el camino, se continuará siempre con una salud débil que no deje avanzar hacia el objetivo común: mejorar la calidad de la enseñanza.

\section{Limitacionesy prospectiva de la propia investigación}

Cada investigación genera unas limitaciones que en ningún caso tendrían que afectar al buen desarrollo de esta. En nuestro caso, las limitaciones que se encontraron fueron:

La falta de literatura y actualizada referente a la comunidad de Catalunya en los dos conceptos a investigar como son la autonomía de centros y el cuerpo de la Inspección educativa. Se añade que a más la mayoría de estudios sobre la Inspección educativa está realizada por los propios Inspectores, la mayoría con falta de experiencia en investigación educativa. Muchos de los artículos analizados se referían a reflexiones y opiniones sobre la práctica de la Inspección educativa.

El proceso de recogida de la información coincidió con la publicación del concurso de oposiciones para el acceso a la Inspección educativa. Este hecho supuso muchas dificultades para responder al cuestionario y realizar las entrevistas previstas 
Referente al proceso metodológico, se asumió las limitaciones propias que representa la metodología cualitativa, del paradigma interpretativo y del método de estudio de casos. Se entiende que los resultados obtenidos y sus conclusiones se aproximan al máximo a la realidad que se pretende, pero nunca a realidad absoluta. La parte cuantitativa a través del cuestionario buscaba una mayor objetividad equilibrando la información cualitativa recogida.

El estudio ha analizado muchos elementos relacionados y vinculados con la Inspección educativa. Aun así, muchos de estos no se han podido desarrollar más porque en este caso no era la finalidad, pero si ayuda a una continuidad a futuros estudios sobre la Inspección educativa, entre estos se proponen 4:

Un estudio donde se recoja las propuestas que debería incorporar un nuevo Decreto que regule la Inspección educativa, ya que el ultimo data del año 2000. Uno de los objetivos de la Inspección educativa es utilizar un modelo menos burocrático de sus tareas. Una propuesta de estudio seria aquel que recoja qué trabajos burocráticos no haría falta desarrollar por parte de la Inspección educativa y que otros agentes pudieran realizarlos.

Una de las carencias detectadas en esta investigación es la falta de aportación a la Innovación educativa. Qué tipo de formación debería hacer, como tendría que ser esta implicación en los centros educativos y como se debería realizar el proceso de asesoramiento seria algunos de los elementos a estudiar. Se plantea una investigación que genere un documento para evaluar la Inspección educativa, entendiendo que esta evaluación ayude a mejorar el cuerpo de la Inspección educativa.

\section{Referencias}

Artigal, R. y Rios, J. (2011). Escola i inspecció en el segle XXI. Fórum. Revista d’Organització i Gestió Educativa, 25, 15-18.

Bolívar, A. (1994). Autonomía escolar en el desarrollo curricular: Razones y problemas. Autonomía institucional de los centros educativos. Universidad de Deusto.

Bolívar, A. (2010). La autonomía de los centros educativos en España. CEE Participación Educativa, 13, 8-25.

Camacho, A. (2015). La visita de inspección, su función malherida. REICE. Revista Iberoamericana sobre Calidad, Eficiencia y Cambio en Educación, 13(4), 79-91.

Castán, J. L. (2016). El reto de la inspección de educación en España. Avances en Supervisión Educativa, 25, 1-41.

Colom, M. M. (2011). El paper de la inspecció en el marc del nou decret d'autonomia. Fòrum. Revista d'Organització $i$ Gestió Educativa, 25, 13-14.

Del Pozo, A. (2016a). El sistema educatiu del segle XXI en un món líquid. Universitat de Lleida.

Del Pozo, A. (2016b). Present i futur de la gestió del professorat a Catalunya. Fòrum. Revista d'Organització i Gestió Educativa, 39, 4-15.

Estañán, S. (2014). Inspección y mejora del sistema. Cuadernos de Pedagogía, 441, 74-77.

Estruch, J. (2006). Autonomía escolar ¿para qué? Cuadernos de Pedagogía, 362, 104-109.

Estruch, J. (2009). Autonomía de centros, tantas oportunidades como riesgos. IDEA, 31, 4-6.

Gairín, J. (2005). La descentralización educativa: ¿Una solución o un problema? Praxis.

Galicia, F. J. (2016). ¿Puede la inspección abordar nuevos retos con su enfoque actual?. Avances en Supervisión Educativa, 25, 7-20.

García Andreu, M. (2013). La autonomía de los centros. Un debate abierto. Revista Supervisión 21, 30, 14-20.

Glaser, B. y Strauss, A. (1967). The discovery of grounded theory. Aldine Press.

Graells, J. (2010). Les dues vessants de l'autonomia escolar: Projecte educatiu i direcció. Guix: Elements D'acció Educativa, 365, 11-15.

Graells, J. y García, I. (2010). El decreto de autonomia de los centros y la mejora de los resultados. Departament d'Educació de la Generalitat de Catalunya.

Hernández Sampieri, R., Fernández, C. y Baptista, M. P. (2010). Metodología de la investigación. McGraw-Hill. 
Jiménez, J. (2016). Una nueva y vieja inspección. Cuadernos de Pedagogía, 441, 58-62.

Larruzea, G. (2011). La autonomía de los centros de la escuela pública vasca: Análisis y propuestas en clave de servicio público y equidad (Tesis doctoral). Universidad del País Vasco.

Lorente, A. (2010). Supervisión educativa: Órgano de la administración educativa. UNED.

Marchesi, A. (2004). La autonomía de los centros docentes. Riesgos, temores y posibilidades. En A. García Albadalejo (Coord.), La autonomía de los centros escolares (pp. 29-63). Ministerio de Educación, Subdirección General de Información y Publicaciones.

Moreno Guerrero, A. (2019). Estudio bibliométrico de la producción científica sobre la inspección educativa. REICE. Revista Iberoamericana sobre Calidad, Eficacia y Cambio en Educación, 17(3), 73-89.

https://doi.org/10.15366/reice2019.17.3.002

Moreno Guerrero, A., Romero Rodríguez, J., Ramos Navas-Parejo, M. y Alonso García, S. (2019). Análisis bibliométrico sobre inspección educativa en la base de datos web of science. REICE. Revista Iberoamericana sobre Calidad, Eficacia y Cambio en Educación, 18(1), 83-103.

https://doi.org/10.15366/reice2020.18.1.005

OCDE. (2014). Programa para la evaluación internacional de alumnos. PISA, 2012, resultados España. OCDE.

Onwuegbuzie, A. J. y Leech, N. L. (2006). Linking research questions to mixed methods data analysis procedures. Qual Report, 11(3), 474-498.

Pérez, A. (2011). La autonomía de los centros sitúa a la inspección educativa en un marco diferente. Avances en Supervisión Educativa, 15, 1-4.

Prat, J. (2011). La nova relació entre la inspecció i les direccions dels centres. Fórum. Revista d'Organització i Gestió Educativa, 25, 19-21.

Sandín, M. P. (2003). Investigación cualitativa en educación. Fundamentos y tradiciones. McGraw-Hill.

Secadura, T. (2013). El referente de la inspección educativa: ¿El centro docente? ¿El sistema educativo? Revista Supervisión 21, 30, 7-21.

Segura, J. (2018). Implicacions per a la inspecció educativa a Catalunya del desenvolupament de l'autonomia de centres (Tesis doctoral). Universitat Autònoma de Barcelona.

Silva, P. (2008). La inspección escolar en Cataluña. Un estudio de casos (Tesis doctoral). Universitat de Barcelona.

Silva, P. (2011). La inspecció escolar i l'autonomia dels centres educatius. Fòrum. Revista d'Organització i Gestió Educativa, 25, 22-24.

Silva, P. (2013). El papel de la inspección escolar en la mejora de los resultados educativos. Educar, 49, 67-82.

Taylor, S. J. y Bogdan, R. (2010). Introducción a los métodos cualitativos. Book Print.

Torres Rubio, F. (2010). Autonomía de centros. ¿Qué autonomía? ¿Cuánta autonomía? Participación Educativa, 13, 104-115.

Vera Mur, J. M. (2014). Supervisión e inspección, a través del tiempo. Cuadernos de Pedagogía, 441, 63-66.

\section{Breve CV de los/as autores/as}

\section{Joan Segura}

Profesor asociado en el departamento de Pedagogía de la Universidad de Lleida. Doctor en educación. Entre los títulos más significativos se encuentran diplomado magisterio en educación musical y educación física, licenciado en Psicopedagogía y Máster en recerca educativa. Ha ejercido de maestro en educación física en la escuela pública y de orientador educativa. Sus ámbitos de especialización son la educación inclusiva y la didáctica de la educación. Email: j.segura@pip.udl.cat

ORCID ID: https://orcid.org/0000-0002-2521-4326 


\section{Joaquín Gairín}

Catedrático de Pedagogía en la Universidad Autónoma de Barcelona (UAB), ha sido decano de centro e instituto universitario, director de departamento y. comisionado para el Clúster de educación y formación en la UAB. Dirige actualmente el Equipo de Desarrollo Organizacional (EDO: http://edo.uab.es), la Red Internacional de Apoyo a la Gestión Educativa (redAGE: http://www.redage.org) y el proyecto europeo ORACLE (https://observatorio-oracle.org/). Sus ámbitos de especialización son: la evaluación y la organización/dirección de instituciones educativas, la gestión del conocimiento colectivo (sistemas educativos, administración pública y empresas) y temas relacionados con las comunidades formativas y de aprendizaje. Email: joaquin.gairin@uab.es

ORCID ID: https://orcid.org/0000-0002-2552-0921

\section{Patricia Silva}

Profesora Agregada del Departamento de Pedagogía de la Universitat de Lleida. Doctora en Pedagogía por la Universitat de Barcelona. Entre los títulos más significativos se encuentran Máster en Dirección de Centros Educativos por la Universidad de Barcelona; Máster en Desarrollo Educativo; licenciada en Sociología y maestra de educación primaria por la Benemérita Escuela Nacional de Maestros. Ha ejercido como Maestra de primaria, asesora escolar y profesora universitaria. Sus ámbitos de especialización son la inspección educativa y la didáctica educativa. Email: patricia.silva@udl.cat

ORCID ID: https://orcid.org/0000-0001-5651-296X 